\title{
Turismo voluntário e o bem-estar do consumidor na pesquisa transformativa do consumo
}

\author{
Volunteer tourism and consumer well-being in \\ transformative consumer research
}

\author{
Thiago Gomes de Almeida ${ }^{1}$ \\ Letícia Moreira Casotti ${ }^{2}$
}

\section{Resumo}

A pesquisa transformativa do consumo é uma vertente de estudos preocupada em contribuir para o bem-estar do consumidor, tendo em vista o impacto gerado pelas práticas de mercado. Ao mesmo tempo, foi possível localizar um referencial também recente sobre turismo voluntário, que envolve lazer e trabalho em prol de auxiliar comunidades em situação de fragilidade, e tem mobilizado esforços de estudos acadêmicos e de indivíduos engajados em questões relacionadas à redução da pobreza e da desigualdade social. Este artigo tem como objetivo analisar o fenômeno do turismo voluntário pelas lentes da abordagem transformativa, destacando como esta abordagem pode contribuir para o desenvolvimento da pesquisa sobre o tema, e como o fenômeno do turismo voluntário pode oferecer oportunidades de teorização para a pesquisa transformativa. Foram identificadas e articuladas três oportunidades de construção de teorias: teorização em nível metodológico, multicultural e em serviços transformativos. Nas considerações finais, são apresentados caminhos futuros de pesquisa no contexto brasileiro.

Palavras-chave: Turismo voluntário. Pesquisa transformativa. Consumo.

\section{Abstract}

The aim of the branch of studies known as Transformative Consumer Research is to contribute to consumer well-being through market practices. In line with this concern

Doutorando em Administração pelo Instituto COPPEAD / UFRJ e Professor da graduação e pósgraduação da ESPM Rio - Brasil - E-mail: thiagogda@oi.com.br

2 Doutorado em Engenharia de Produção, UFRJ. Pesquisadora e Professora do Programa de Pós-graduação em Administração do Instituto COPPEAD / UFRJ - Brasil - E-mail: leticia@ coppead.ufrj.br 
a considerable body of recent research has focused on volunteer tourism. This involves leisure and work activities aimed at helping communities in a fragile situation and has mobilized the efforts of academic studies and individuals engaged in issues related to the reduction of poverty and social inequality. The aim of this article is to analyze the phenomenon of volunteer tourism through the lens of the transformative approach, highlighting the way it can contribute to the development of research into the theme and how the volunteer tourism phenomenon can provide theorization opportunities for transformative research. The study identified and articulated three opportunities for the construction of theories, at the methodological and multicultural levels and in transformative services. Suggestions for future paths of research in the Brazilian context are presented in the final considerations.

Keywords: Volunteer Tourism.Transformative Research. Consumption.

\section{Introdução}

Desde os anos 1960, a pesquisa em marketing vem demonstrando crescente preocupação com a questão do desenvolvimento social, econômico e ambiental, e principalmente com o bem-estar do consumidor (Andreasen et al., 2012; Hilton, 2008; McGregor, 2010; Speth, 2008; Wilkie \& Moore, 1999, 2003, 2012). Um importante sinal de amadurecimento dessa preocupação foi quando, em 2006, a Association of Consumer Research, por meio de seu então presidente, David Glen Mick, apresentou uma comunicação na qual sugere a organização de uma nova vertente de estudos em consumo, chamada de Transformative Consumer Research (TCR), ou pesquisa transformativa do consumo (MICK, 2006). Com o foco e compromisso em promover uma pesquisa que contribua diretamente para a transformação positiva e para a melhoria do bem-estar e das condições de vida do consumidor, a TCR se propõe a investigações de temas como a obesidade, consumo de tabaco, álcool e drogas, consumo de produtos financeiros, consumo de jogos e apostas, restrições à alimentação saudável e serviços sociais e de saúde (MICK et al., 2012).

O compromisso central da TCR com as implicações práticas deu origem a interpretações equivocadas de que essa vertente de estudos não teria capacidade ou interesse na construção de teorias. Pesquisadores 
da TCR, no entanto, buscam desconstruir essa suposição (Mick et al., 2012; Ozanne, 2011; Ozanne \& Anderson, 2010). A percepção de que os trabalhos da perspectiva transformativa não teriam potencial teórico é prejudicial para o desenvolvimento do campo, e também imprecisa, uma vez que as perspectivas ontológicas que fundamentam essa abordagem são ancoradas no paradigma transformativo, que estabelece como objetivo a prática de uma ciência social que construa conhecimento teórico que impacte positivamente no bem-estar das sociedades (MERTENS, 2007; 2010).

Ao mesmo tempo em que se desenvolve o arcabouço da TCR, surge no campo do turismo um novo fenômeno, classificado como turismo voluntário (WEARING, 2001), que vem despertando interesse da comunidade acadêmica e originando publicações sobre o tema (MCGEHEE, 2014). O turismo voluntário foi primeiro conceituado por Wearing (2001) como uma prática em que turistas viajam para voluntariar durante feriados e férias, em ações de curto prazo, que envolvem contribuições para alívio e diminuição da pobreza de grupos sociais em situação de fragilidade, e apoio na restauração e manutenção do meio ambiente. O potencial transformativo do turismo voluntário já foi detectado pela literatura, por meio dos trabalhos que apresentam relatos sobre as transformações positivas que acontecem na vida dos voluntários após a experiência, e nos impactos gerados para as comunidades locais que recebem o benefício do serviço voluntário (MCGEHEE, 2014; MCINTOSH \& ZAHRA, 2007).

Este ensaio teórico procura demonstrar atributos e elementos do turismo voluntário que o caracterizam como um fenômeno relevante para a investigação da abordagem transformativa em consumo. Esta relevância se daria em duas dimensões: a primeira, pelo potencial de geração de bem-estar do turismo voluntário para os consumidores, tanto os que o praticam, quanto os que recebem seu benefício. A segunda, pelas oportunidades que o fenômeno oferece em termos de teorização para a abordagem transformativa. Ao mesmo tempo, procura também discutir possibilidades de contribuição dessa abordagem para 
a potencializar elementos positivos do turismo voluntário, e ao mesmo tempo conter impactos negativos gerados pela sua prática.

Este trabalho está dividido em quatro partes, iniciando-se com a discussão sobre a ontologia e a epistemologia da pesquisa transformativa em consumo, com destaque para a questão do processo de teorização dentro desta abordagem. Na segunda parte, é discutido o fenômeno do turismo voluntário e como a área de conhecimento ligada ao turismo tem desenvolvido seu tema. Na terceira parte, são apresentados os argumentos que justificam a relevância da pesquisa do fenômeno do turismo voluntário sob as lentes da abordagem transformativa e seu potencial de teorização. Na quarta e última parte, são realizadas considerações finais e indicados caminhos futuros para a pesquisa sobre o tema.

\section{Pesquisa transformativa do consumo: ontologia e contribuições teóricas}

A TCR tem como preocupação central promover uma pesquisa que contribua para a melhoria do bem-estar e da qualidade de vida do consumidor e da sociedade em geral, mediante práticas de mercado. Mick et al. (2012) propõem quatro visões para o desenvolvimento da TCR: 1) estratégias alternativas de comunicação, que significa a busca por modos de disseminação dos resultados de pesquisa que ampliem seus potenciais de aplicação junto à sociedade; 2) agentes de mudança social, relacionado principalmente com a postura do pesquisador e seus relacionamentos com os contextos pesquisados; 3) a sabedoria prática, que se trata da fundamentação filosófica da abordagem transformativa; e 4) contribuições teóricas, que dizem respeito ao processo de teorização que deve ser promovido pelos pesquisadores desta vertente. As visões sobre a sabedoria prática e contribuições teóricas, são aquelas que constituem o foco principal deste ensaio.

Para iniciar a compreensão em relação à sabedoria prática como noção incorporada pela TCR, partimos do resgate da obra de Aristóteles, 
realizada pela filosofia das ciências sociais (FLYVJBERG, 2004). Em Ética à Nicômano, Aristóteles apresenta os três saberes ou virtudes intelectuais, que devem ser buscadas pelo homem: o saber teórico da epistéme, o saber fazer da techné e o saber moral ou sabedoria prática da phronesis (MAXWELL, 2007). A epistéme foi definida pelo filósofo como sendo a virtude do conhecimento científico. A techné seria a virtude do saber fazer, orientada a um objetivo específico. Já a phronesis, ou sabedoria prática, foi classificada por Aristóteles como a principal virtude intelectual, responsável por balizar os julgamentos éticos do indivíduo, ou seja, de nada adiantaria, por exemplo, um sujeito possuir a virtude técnica bem desenvolvida, se esta não fosse aplicada para o bem social comum.

A noção de sabedoria prática originada da filosofia será recuperada por Flyvbjerg (2004) e Maxwell (1984, 2007, 2009), que propuseram uma nova perspectiva para a filosofia das ciências sociais, denominada Phronetic Planning Research (PPR). Sua ontologia argumenta em favor de uma prática da pesquisa social que promova contribuições concretas para o bem-estar da sociedade. Esta visão se opõe a uma noção de prática científica que privilegia demasiadamente a construção de teorias incapazes de atravessar as fronteiras do universo acadêmico, diminuindo consideravelmente o potencial de tais teorias em impactar positivamente a vida social (FLYVBJERG, 2004).

A perspectiva da pesquisa transformativa nas ciências sociais é intrinsecamente relacionada com a noção de bem-estar e equilíbrio social, a partir de uma lógica que compreende a pesquisa científica como instrumento de contribuição para a melhoria da qualidade de vida e bem-estar da sociedade e procura pensar as relações de poder que se configuram nesse contexto (MERTENS, 2010). Mertens (2007) apresenta algumas questões que tipicamente organizam a ontologia do paradigma transformativo: como a realidade é definida? Por quem? Quem é privilegiado por esta definição? Quais as implicações em termos de justiça social em se aceitar tal definição da realidade sem uma discussão crítica? Para Mertens (2007), esses questionamentos de ordem ontológica são a base para uma epistemologia do paradigma 
transformativo na sociologia, que preconiza o relacionamento de parceria do pesquisador com as comunidades e seus membros, permitindo a identificação das diferenças de poder, bem como de construção de elos de confiança entre ambos. A metodologia neste paradigma deve considerar não apenas a conscientização sobre a multiplicidade de realidades sociais, como também incorporar a comunidade durante o processo de pesquisa.

Mick et al. (2012), ao apresentarem as visões que norteiam a prática da TCR, apropriaram-se da proposta da PPR de Flyvbjerg e Maxwell, partindo de sua ontologia para construir uma aplicação desta aos estudos de consumo. Também procuraram sinalizar os temas de maior sensibilidade, no que se refere ao bem-estar do consumidor no contexto de mercado contemporâneo, como obesidade, consumo de álcool, vícios em geral, tabaco, violência e redução da pobreza.

De um ponto de vista epistemológico, a TCR se articula a partir de fortes influências da pesquisa de ação participativa (OZANNE \& FISCHER, 2012; OZANNE \& SAATCIOGLU, 2008), a qual preconiza uma pesquisa acadêmica que promova a mudança social, mediante uma agenda de pesquisa que incorpore os interesses dos grupos sociais tradicionalmente marginalizados, e de uma ação do pesquisador no campo que revele as perspectivas e interesses desses grupos. Outras modalidades também são destacas por Mick et al. (2012), como: pesquisa de revelação, que busca desvendar dimensões da vida social, normalmente relativa a grupos em situação vulnerável, não conhecidas pelo grande público, chamando atenção da mídia e autoridades para o problema em questão, buscando conhecer a perspectiva dos indivíduos inseridos em tal realidade; pesquisa política, que dá atenção a como leis e políticas públicas influenciam o bem-estar dos cidadãos; pesquisa de coalisão, na qual o pesquisador, ao aderir pessoalmente a determinada causa ou grupo social, decide construir um relacionamento com indivíduos pertencente a esse grupo, com o objetivo de gerar pesquisas adicionais e mais auxílio ao território em questão; por fim, a pesquisa incendiária, na qual o pesquisador busca ir além das fronteiras acadêmicas para 
apresentar suas ideias ao público em geral, fomentando o debate que permeia sua pesquisa.

As instruções sobre a epistemologia da TCR demonstram como ela incorpora a noção de sabedoria prática da PPR, tanto pela definição de seus temas, quanto pela maneira como buscará construir conhecimento. No entanto, essa perspectiva participativa, crítica e orientada para a prática, que caracteriza a TCR, tem sido avaliada por parte da comunidade acadêmica que pesquisa o consumo, como tendo uma capacidade limitada para criar e desenvolver teoria (Mick et al., 2012). O campo do comportamento do consumidor já debate há tempos a questão do desenvolvimento de teorias (Belk, Sherry \& Wallendorf, 1988; Bringberg \& Hirschman, 1986; Holbrook, 1985; Hudson \& Ozanne, 1988; Hunt, 1991) e sua importância para o avanço e consolidação da perspectiva científica dos estudos de consumo. No entanto, também se registrou um intenso debate sobre o abismo que constituiu entre as pesquisas focadas no desenvolvimento de teoria e as pesquisas substantivas, orientadas para as questões do bem-estar e qualidade de vida, dentro do campo (Bringberg \& Hirschman, 1986). Para Mick et al. (2012), o caráter substantivo da TCR não implica na impossibilidade de teorização. Parte desta percepção se daria pela maneira como a pesquisa nesta vertente se constitui.

$\mathrm{Na}$ TCR, todo processo de pesquisa parte de uma realidade em que se observa determinado problema no âmbito socioeconômico, e a articulação de teorias se dá posteriormente, servindo de guia para o trabalho do pesquisador. Dessa maneira, as teorias são utilizadas como meios para se obter um fim que, normalmente, se constitui em contribuição para mudanças sociais. Durante esse processo, a teorização acontece como consequência da abstração que o pesquisador realiza a partir de seu trabalho de campo, portanto, ela é consequência, e não alvo. Mesmo que posicionada de forma menos destacada na proposta da TCR, o desenvolvimento de teoria acontece e é praticado pelo pesquisador. Mick et al. (2012) citam o exemplo de Marcoux (2009), que por meio de seu trabalho trouxe novas perspectivas sobre os impactos 
negativos da pesquisa em gift-giving, até então não observados pelos estudos de consumo de abordagem tradicional.

Mick et al. (2012) prescrevem quatro etapas para um processo de desenvolvimento de pesquisa transformativa que contemple a teorização. A primeira etapa seria a conceptualização de um problema substantivo em um nível mais alto de abstração. Ou seja, apesar de o objeto de estudo ser oriundo do mundo social, e de os objetivos de tal estudo serem melhorar a condição de bem-estar dessa respectiva faceta da vida social, a pergunta de pesquisa deve remeter a um grau maior de abstração, procurando por uma generalização teórica, aplicável ou crível para outros contextos. Sendo assim, a resposta a essa pergunta, ao mesmo tempo atenderia aos parâmetros teóricos, e estaria dirigida diretamente à resolução de um problema específico da realidade social. Em segundo lugar, Mick et al. (2012) recomendam que o pesquisador encontre uma aderência entre sua pergunta de pesquisa e construtos e teorias pré-existentes, que possam ajudá-lo a estruturar seu trabalho. Esse processo se daria fundamentalmente por meio de revisão de literatura, que preferencialmente seria conduzida em uma perspectiva multidisciplinar. Na terceira etapa, o pesquisador deveria construir hipóteses para testar as teorias e em sequência verificar as possíveis contribuições teóricas. Por fim, na quarta etapa, as hipóteses deveriam ser testadas e seus construtos teóricos enfatizados em termos de contribuição teórica.

Seguindo as instruções de Mick et al. (2012) sobre a construção de conhecimento teórico na TCR, a seguir será analisado o fenômeno do turismo voluntário e como ele vem sendo analisado por pesquisadores de da área de turismo. Considera-se aqui que esse pode ser um contexto de pesquisa relevante para TCR, tanto pela sua capacidade de geração de impacto positivo no bem-estar da sociedade quanto pelo seu potencial de contribuição teórica para a TCR. 


\section{Turismo voluntário: origem, conceito e experiência transformadora}

O turismo é uma atividade social cujo amadurecimento comercial tem produzido grande diversificação e sofisticação do produto turístico, entre as quais se destaca o segmento do turismo alternativo (Barbieri et al., 2012). Essa modalidade turística é caracterizada por pequena escala e é centrada na dimensão humana, guiada por valores de comunidade e para o benefício tanto dos hóspedes e anfitriões como do ambiente em que a prática se dá (Lyons \& Wearing, 2008; Mcgehee, 2009). Uma das vertentes que compõe o turismo alternativo é o turismo voluntário (Barbieri et al., 2012), definido como uma atividade que envolve viagem, prazer e recreação, com o trabalho voluntário em benefício de uma determinada comunidade ou grupo social (Wearing, 2001; Rayomnd, 2008; Barbieri et al., 2012; Mcgehee, 2014).

A pesquisa sobre o turismo voluntário tem demonstrado que essa atividade envolve não apenas a dimensão de diversão e ou experiências exóticas para o participante, mas principalmente um desejo de contribuir para o alívio da pobreza e geração de melhorias sociais e das condições de vida das populações dos territórios onde sua prática acontece (Lyons \& Wearing, 2008; Mcgehee \& Santos, 2005).

O fenômeno do turismo voluntário tem ganhado cada vez maior relevância e espaço, no que se reflete tanto ao crescimento da produção acadêmica sobre o tema, quanto em sua prática em nível global e sua presença na mídia de massa (Wearing \& Mcgehee, 2013). Elliot (2008 apud Mcgehee, 2014) identificou que a participação de pessoas em atividades de turismo voluntário e a pesquisa sobre o tema cresceu exponencialmente nos últimos 20 anos. Estima-se que, em média, desde 1990, aproximadamente 1,6 milhão de pessoas ao redor do mundo participa anualmente de projetos de turismo voluntário. No entanto, a média dos últimos anos estima algo próximo a 10 milhões de pessoas engajadas nestas atividades anualmente (Wearing \& Mcgehee, 2013). Turistas voluntários gastam em média entre 700 milhões e 1 bilhão de 
euros por ano em suas atividades voluntárias (Tourism Research \& Marketing, 2008).

Lo e Li (2011) explicam que o turismo voluntário possui suas raízes na Grã-Bretanha e Europa ocidental, mas rapidamente se popularizou nos Estados Unidos e Austrália, através do envolvimento da classe média e da comunidade evangélica, para a realização de missões. Alexander (2012) observou o crescimento do turismo voluntário também na Ásia e África. O interesse neste fenômeno posicionou o turismo voluntário como um campo de estudos emergente dentro da pesquisa em turismo e chamou atenção para sua natureza transformativa (Barbieri et al., 2011; Lyons \& Wearing, 2008; Mcgehee, 2009; Mcgehee \& Santos, 2005; Mcintosh \& Zahra, 2007; Sin, 2009; Stoddart \& Rogerson, 2004).

Não existe consenso ainda sobre a definição de turismo voluntário, mas aquela oferecida por Wearing (2001) tem sido a mais comumente utilizada, na qual o autor afirma que "o termo genérico 'turismo voluntário' se aplica a turistas que, por diversas razões, se organizam para voluntariar durante feriados e férias, em ações que costumam envolver contribuições para alívio e diminuição da pobreza de certos grupos sociais, restauração e manutenção do meio ambiente, ou mesmo pesquisa sobre determinados aspectos do mundo social". Argumenta que, apesar do propósito do voluntário ser o de trabalhar durante as férias ou feriados para contribuir com a redução de desigualdades sociais, não existem evidências concretas de que este é sempre o propósito central do praticante do turismo voluntário, o que o diferencia do voluntário tradicional, que comumente dedica um ano sabático para voluntariar, e não necessariamente se desloca de sua comunidade original. Para Butcher e Smith (2010), uma das razões do expressivo crescimento desta atividade é a procura dos indivíduos, com destaque para jovens adultos, pela construção de suas identidades através da busca por fazer a diferença no mundo.

Coghlan (2007) destaca o papel dos operadores, ou facilitadores, de turismo voluntário, para o desenvolvimento da atividade. Instituições não governamentais, empresas privadas, ou mesmo grupos de 
indivíduos oriundos da sociedade civil, têm sido responsáveis pela organização sistemática de iniciativas de turismo voluntário. Um exemplo é a Earthwatch, organização internacional de destaque na operação de turismo voluntário, que entre 1971 e 2008 trabalhou com mais de 90.000 voluntários, em 1.350 diferentes projetos em 120 países (Earthwatch Institute, 2015). Wearing e McGehee (2013) demonstraram que a busca pelo termo "Volunteer Tourism" no Google, em 17 de abril de 2008, retornou 230.000 resultados. A mesma busca quatro anos depois, em 17 de abril de 2012, retornou 4.850.000 resultados. A indústria do turismo voluntário também apresenta pujança. Callanan e Thomas (2005) identificaram 698 produtos de turismo voluntários diferentes apenas em um site dedicado ao tema (goabroad.com). Essa atividade de operação e intermediação tem sido compreendida pela literatura (Barbieri et al., 2011) como relevante tanto para o desenvolvimento pessoal do voluntário, como para o da comunidade onde este atua (Wearing et al., 2005). O turismo voluntário trabalha com a premissa de que todos os envolvidos no processo devem ser beneficiados.

Do ponto de vista do voluntário, a literatura relata essa experiência como sendo transformadora para os indivíduos que passam por ela (Zahra \& Mcintosh, 2007). O voluntário investe tempo, recursos financeiros e trabalho para contribuir com o desenvolvimento de uma determinada comunidade estrangeira. A recompensa recebida por esse investimento advém do caráter transformador da experiência do turismo voluntário, no sentido de provocar mudanças de valores, consciência social, visão de mundo e estilo de vida. O gatilho gerador de tais transformações individuais está intrinsecamente relacionado com a interação do voluntário com todos os fatores do trabalho de campo, principalmente o contato próximo com a população que recebe a ajuda do voluntário, e sua percepção sobre o impacto positivo que seu trabalho foi capaz de gerar (Coghlan, 2007; Mcgehee, 2015; Wearing, 2001; Wearing, 2002; Wearing et al., 2005; Zahra \& Mcintosh, 2007) argumento que a experiência de conhecer e aprender sobre outras pessoas e culturas a partir da interação do trabalho de campo, em um ambiente 
de colaboração e cooperação, é o que permite o desenvolvimento e transformação individual.

Zahra e McIntosh (2007), ao realizarem um estudo de caso de turismo voluntário na comunidade Maori, identificaram que as vivências culturais e interações que os voluntários obtiveram no campo foram genuínas, reflexivas, ricas em autenticidade cultural, e promoveram efeitos catárticos nas vidas dos voluntários participantes. Stoddart e Rogerson (2004) identificaram diferenças entre o turista voluntário e o turista tradicional, observando que os voluntários buscam relações pessoais de proximidade com a população local, o que promove experiências que mesclam valores socioculturais, gerando oportunidades de desenvolvimento individual e altruístico. As principais categorias de turismo voluntário foram identificadas nas áreas de restauração histórica e cultural, assistência médica, educação, conservação ecológica, e contribuições para o meio ambiente (Coghlan, 2008; Coghlan \& Fennel, 2009; Cousins, 2007; Cousins, Evans, \& Sadler, 2009; Gray \& Campbell, 2007; Rattan, Eagles, \& Mair, 2012).

Andreasen et al. (2012), ao relatarem a trajetória e evolução do conceito e pesquisa em marketing social ao longo da segunda metade do século $X X$, diferenciam os trabalhos publicados a partir de duas dimensões, classificadas como marketing's dark side e marketing's bright side, aqui traduzidas como o lado obscuro do marketing e o lado positivo do marketing, respectivamente. O lado obscuro do marketing seria responsável por estudos que tem como interesse mitigar os efeitos negativos da prática de mercado, como as questões de produtos de causam vício, endividamento financeiro e desigualdade social (Caplovitz, 1963), por exemplo. O lado positivo do marketing concentra os estudos que evidenciam o potencial do marketing para contribuir com o bemestar, como pesquisas sobre eficiência e gestão de organizações sem fins lucrativos, e as políticas de distribuição de produtos e serviços básicos para as populações em situação de fragilidade (Kolter \& Levy, 1969). 
Nesse sentido, a caracterização apresentada sobre o fenômeno do turismo voluntário apresenta fortes elementos de aderência com as lentes teóricas da pesquisa transformativa, tanto pela perspectiva do lado obscuro, quanto do lado positivo do marketing. Além disso, é preciso considerar o fato de que a prática do turismo voluntário é um ato de consumo, uma vez que o voluntário realiza o pagamento de seu programa para ter acesso à experiência, o que também se constituí num tema de interesse para a abordagem transformativa. No próximo tópico, esses movimentos de aproximação serão explorados com maior detalhamento, bem como a descrição das oportunidades de teorização que o fenômeno pode oferecer à TCR.

\section{Turismo voluntário e pesquisa transformativa do consumo: possibilidades e aproximações}

Pesquisar o turismo voluntário pela abordagem transformativa do consumo é um movimento que a priori se conecta as questões ontológicas e epistemológicas colocadas pela literatura (Mcgehee, 2014; Mick et al., 2012). Do ponto de vista ontológico, a aproximação entre o fenômeno e a TCR se dá pelo objetivo comum do qual ambos comungam: contribuir para uma mudança social positiva e melhoria da qualidade de vida dos grupos sociais em situações de fragilidade, partindo da crença de que a realidade social é fragmentada e portanto o auxílio às camadas menos favorecidas deve acontecer de modo independente das ações governamentais. O turismo voluntário busca alcançar esse objetivo através da criação e execução de seus programas, gerenciados por operadores e facilitadores (Mcgehee, 2014). A TCR, por sua vez, através da pesquisa acadêmica de impacto prático.

A PPR, ao incorporar a discussão de poder em sua proposta (Flyvbjerg, 2004), sinaliza preocupação em trazer à tona dos debates sociais e da pesquisa social, a visão de mundo e demandas da parcela da sociedade menos favorecida. Essa noção está claramente assumida e incorporada pela TCR, e pode ser observada também no turismo voluntário. $\mathrm{O}$ indivíduo que toma a decisão de dedicar parte 
de seus recursos financeiros e tempo livre para a realização de ajudas humanitárias e ao meio ambiente, está, de certa maneira, imbuído do mesmo espírito que caracteriza a pesquisa na TCR. O ponto de partida parece ser o mesmo: o pressuposto de que há desigualdades que precisam ser atenuadas para que contingentes de população passem a gozar de uma melhor condição de bem-estar e qualidade de vida. Mesmo que a pesquisa em turismo não anuncie um compromisso inicial com as implicações práticas no sentido extremo que a TCR se propõe a fazer, há reconhecimento da comunidade acadêmica de que o fenômeno do turismo voluntário resgatou a dimensão do potencial social da atividade turística no campo (Barbieri et al., 2012; Mcgehee, 2014; Mcgehee \& Santos, 2005; Wearing et al., 2005).

Se as similaridades ontológicas são objetivamente identificadas, do ponto de vista epistemológico há diferenças importantes. A pesquisa sobre esse fenômeno no campo de turismo se caracteriza por um viés descritivo e ideológico (Mcgehee, 2014). Dessa maneira, a perspectiva transformativa pode oferecer uma importante contribuição: acrescentar à descrição e à ideologia uma dimensão prática, de impacto direto na realidade social, conforme a preconizam Ozzane e Saatcioglu (2008) e Mick et al. (2012). A relevância da perspectiva epistemológica da TCR se mostra ainda maior quando se trata dos impactos negativos do turismo voluntário, observados pela literatura (Guttentag, 2009). Se a prática dessa atividade tem como riscos potenciais a redução de empregos, geração de dependência por parte seus beneficiados, ou mesmo fomento a um processo de imposição de valores socioculturais por parte dos voluntários, ou seja, impactos práticos, uma abordagem como a transformativa pode contribuir através de pesquisas que auxiliem na criação de soluções que mitiguem tais impactos, uma vez que há evidências dos benefícios do turismo voluntário, tanto para o voluntário quanto para a população local (Mcgehee, 2014).

As possibilidades metodológicas destacadas por Mick et al. (2012), como a pesquisa incendiária, de coalisão e política, e também propostas por Ozanne e Fischer (2012), como a ação participativa e a participação comunitária (Ozzane \& Saatcioglu, 2008), podem ter forte aderência 
ao estilo de investigação que o turismo voluntário demanda. Portanto, retomando Andreasen et al. (2012), se pelo lado obscuro do marketing a TCR pode contribuir com a mitigação dos efeitos negativos do turismo voluntário, pelo seu viés epistemológico, pode também contribuir pelo lado positivo do marketing, na identificação e potencialização de práticas que fomentem os efeitos positivos desta atividade, tanto para o voluntário quanto para a população beneficiada pelo voluntariado.

Com relação às oportunidades que o turismo voluntário oferece para a construção de teoria no âmbito da TCR, propõe-se três caminhos iniciais, cuja relevância, acredita-se, possa trazer contribuições de curto prazo para o campo. Em primeiro lugar, o turismo voluntário constitui um contexto de pesquisa interessante para a construção de teorias em nível metodológico. A abordagem transformativa se viabiliza e se diferencia, principalmente devido ao seu caráter metodológico, que muitas vezes pressupõe uma participação ativa do pesquisador no campo, bem como suas interações com os indivíduos das comunidades locais. O turismo voluntário, por definição, exige que os voluntários se desloquem de seus territórios de origem e interajam com uma comunidade, normalmente num cenário internacional (Mcgehee, 2014). Os elementos que caracterizam a experiência internacional costumam evidenciar as nuances e traços culturais das comunidades beneficiadas, bem como a distância que as separa das culturas dos voluntários (Zahra \& Mcintosh, 2007). Nesse contexto complexo, os desafios de pesquisa tornam-se de grande envergadura. Ao mesmo tempo em que sugere um campo extremamente rico para o desenvolvimento teórico das metodologias específicas adotadas e sugeridas pela TCR, o fenômeno permite também que uma mesma metodologia seja testada e aplicada simultaneamente em diversas culturas, o que pode trazer benefícios em termos de desenvolvimento teórico e validação de construtos.

Em segundo lugar, o turismo voluntário pode oferecer uma ampliação do escopo de trabalho aos pesquisadores da TCR em termos socioculturais. Os relatos de Andreasen et al. (2012) e Wilkie e Moore (2003) mostram que grande parte dos estudos no âmbito do marketing social estiveram focados historicamente em problemas típicos da 
sociedade norte-americana, como questões raciais, restrição ao acesso a produtos e serviços de maior qualidade pela população de baixa renda, e endividamento e taxas de juros abusivas para comunidades de minorias, como hispânicos, negros e imigrantes em geral. Conforme Barbieri et al. (2012) e McGehee (2014) explicam, o turismo voluntário tem sido praticado primordialmente no continente africano e na Ásia, em um fluxo no qual voluntários oriundos de países desenvolvidos procuram ir trabalhar em regiões subdesenvolvidas ou em desenvolvimento. Portanto, o campo de trabalho do turismo voluntário oferece aos pesquisadores a possibilidade de generalização teórica em âmbito internacional e comparativo. O caráter substantivo, por ora limitador, na visão de parte dos pesquisadores, ganha nova proporção ao passo de que pode ser comparado em diversos contextos culturais.

Por fim, o turismo voluntário pode oferecer um grande e novo espaço de trabalho para a pesquisa em serviços transformativos (Davis, 2013; Rosenbaum et al., 2011). Por se tratar de um fenômeno que não está restrito à perspectiva do consumidor, como McGehee (2014) sugere, boa parte dos estudos tem se concentrado no papel que os facilitadores, ou operadores do turismo voluntário, cumprem, tanto positiva, quanto negativamente, em relação aos resultados que a experiência proporciona aos seus participantes. Os serviços transformativos se apresentam como um promissor e importante campo de estudos dentro da perspectiva da TCR (Anderson et al., 2012), devido ao potencial que serviços em geral possuem para influenciar a qualidade de vida da população.

Segundo Rosenbaum et al. (2011), os serviços transformativos podem ser classificados como aqueles que são intencionalmente projetados para promover o bem-estar e aqueles que são projetados com cunho comercial, mas contêm potencial para promover melhoria na qualidade de vida e bem-estar das populações. O turismo voluntário claramente se configura como um serviço transformativo projetado com a intenção de promover bem-estar. No entanto, nem sempre esse tem sido o resultado alcançado (Guttentag, 2009). A abordagem de serviços pode contribuir para avanços nessa dimensão, assim como novas 
perspectivas teóricas também podem emergir a partir do estudo deste fenômeno.

\section{Reflexões finais}

Procurou-se discutir neste ensaio o potencial de pesquisa que o fenômeno do turismo voluntário possui como objeto de estudo e contexto para a pesquisa transformativa do consumo. A aproximação entre essas duas áreas de conhecimento tornou possível compreender a capacidade de contribuição teórica que o turismo tem a oferecer para o campo da TCR, com destaque para três dimensões: metodológica, multicultural e serviços transformativos.

Os trabalhos influenciados pela vertente transformativa ainda são incipientes no Brasil, a despeito do país representar um contexto rico para esta modalidade de pesquisa, dado o seu caráter de nação em desenvolvimento, conforme atestam Shultze Shapiro (2012). Em verdade, nações como o Brasil e demais que se encontram em desenvolvimento ou subdesenvolvimento se apresentam como contextos extremamente relevantes para a abordagem transformativa. Dessa maneira, este ensaio procurou se articular como veículo de difusão desta perspectiva de estudos no país, de modo a estimular a produção nacional sobre marketing e consumo a partir da abordagem transformativa.

Algumas sugestões de campos de pesquisa são aqui apresentadas:

- Investigar o turismo voluntário no Brasil. Conhecer motivações, práticas e significados do consumo do turismo voluntário para os estrangeiros que contratam os programas, bem como impactos positivos e negativos.

- Investigar a participação do turista voluntário brasileiro no Brasil e em outros países. Conhecer motivações, práticas e significados do consumo do turismo voluntário para os brasileiros que contratam os programas, bem como impactos positivos e negativos. 
- Conhecer a atuação dos operadores/facilitadores de turismo voluntário no Brasil a partir do campo.

- Identificar problemas sociais brasileiros que poderiam receber suporte de programas de turismo voluntário.

- Compreender o contexto e o comportamento da população que recebe esse turismo através da interação e participação no campo.

- Compreender o processo de transformação dos diferentes agentes envolvidos na experiência de turismo voluntário, através da interação e atuação no campo.

Acredita-se que esses contextos de pesquisa possuem potencial para desenvolvimento das três dimensões de construção teórica discutidas por este ensaio. Além disso, essa temática pode fornecer importantes contribuições teóricas para os estudos de consumo e contribuições práticas para diferentes tipos de políticas públicas em todas as regiões brasileiras, do interior aos grandes centros urbanos. Sabe-se também que essa temática e as possibilidades teóricas e metodológicas aqui discutidas podem ser um elo entre o que acontece no mundo, no país, nas cidades de menor porte, e nas comunidades, em geral excluídas da pesquisa de marketing. Os indivíduos têm pouca oportunidade de se informar e de presenciar o que acontece fora do seu cotidiano.

\section{Referências}

Andreasen, A. R., Goldberg, M. E., \& Sirgy, J. (2012). Foundational research on consumer welfare Transformative Consumer Research, New York, NY: Routledge, pp. 25-65

Barbieri, C., Santos, C. A., \& Katsube, Y. (2012, June). Volunteer tourism: On-the-ground observations from Rwanda. Tourism Management, Hamilton, New Zealand, 33(3), 509-516.

Barkin, D. (2009, March) Social tourism in rural communities: An instrument for promoting sustainable resource management. Paper 
presented at Latin American Studies Association Conference, Miami, 1 - 23.

Belk, R. W., Sherry Jr, J. F., \& Wallendorf, M. (1988, March 1). A naturalistic inquiry into buyer and seller behavior at a swap meet. Journal of Consumer Research, Oxford, 449-470.

Bone, S. A., Williams j. D., and Christensen G. L. (2010, June) When Consumer Well-Being Meets Small Business Ownership: Transforming Financial Service Systems to Eradicate Disparate Treatment and Discrimination. In Terri L. Rittenburg and Mark PETERSON (Eds.), The 35th Annual Meeting of the Macromarketing Society: Exploring the Frontiers of Macromarketing, Laramie, WY: Macromarketing Society.

Brinberg, D., \& Hirschman, E. C. (1986, Octuber). Multiple orientations for the conduct of marketing research: An analysis of the academic/ practitioner distinction. The Journal of Marketing, Chicago, 161-173.

Butcher, J., \& Smith, P. (2010). 'Making a difference': Volunteer tourism and development. Tourism Recreation Research, Oxon, UK, 35(1), 27-36.

Caplovitz, D. (1967) The poor pay more. New York: Free Press.

Coghlan, A. (2007). Towards an integrated image-based typology of volunteer tourism organisations. Journal of Sustainable Tourism, New York, 15(3), 267-287.

Coghlan, A. (2008 March/April). Exploring the role of expedition staff in volunteer tourism. International Journal of Tourism Research, Cairns, Australia, 10(2), 183-191.

Coghlan, A., \& Fennell, D. (2009). Myth or substance: An examination of altruism as the basis of volunteer tourism. Annals of Leisure Research, 12(3-4), 377-402.

Coghlan, A., \& Gooch, M. (2011 February). Applying a transformative learning framework to volunteer tourism. Journal of Sustainable 
Tourism, New York, 19(6), 713-728.

Cousins, J. A. (2007 August). The role of UK-based conservation tourism operators. Tourism Management, Edgbaston, Birmingham,UK 28(4), 1020-1030.

Cousins, J. A., Evans, J., \& Sadler, J. P. (2009 November). 'I've paid to observe lions, not map roads!'-An emotional journey with conservation volunteers in South Africa. Geoforum, 40(6), 10691080.

Davis, B., \& Pechmann, C. (2013 August). Introduction to the Special Issue on transformative consumer research: Developing theory to mobilize efforts that improve consumer and societal well-being. Journal of Business Research, Massachusetts, USA, 66(8), 11681170.

Earthwatch institute website (2015) From <http://earthwatch.org/about/ fast-facts\#The-Figures>. Acessed in April, 92015

Elliott, D. (2008) Voluntourism. From <http://www.concierge.com/ cntraveler/articles/12200>. Acessed em 04.05.2015.

Flyvbjerg, B. (2004). Phronetic planning research: Theoretical and methodological reflections. Planning Theory \& Practice, London, 5(3), 283-306.

Gray, N. J., \& Campbell, L. M. (2007). A decommodified experience? Exploring aesthetic, economic and ethical values for volunteer ecotourism in Costa Rica. Journal of Sustainable Tourism, New York, 15(5), 463-482.

Guttentag, D. A. (2009 November/December). The possible negative impacts of volunteer tourism. International Journal of Tourism Research, Waterloo, Ontario, Canada, 11(6), 537-551. DOI: 10.1002/ jtr.727

Hilton, M. (2008) Prosperity for all: Consumer activism in an era of globalization. Ithaca, NY: Cornell University Press. 
Holbrook, M. B. (1985). Why business is bad for consumer research: the three bears revisited. Advances in consumer research, Duluth, MN, 12(1), 145-156.

Hudson, L. A., \& Ozanne, J. L. (1988 March). Alternative ways of seeking knowledge in consumer research. Journal of Consumer Research, Oxford, 14(4), 508-521. DOI: 10.1086/209132

Kotler, P., \& Levy, S. J. (1969). Broadening the concept of marketing. The Journal of Marketing, Chicago, 33(1), 10-15. DOI: 10.2307/1248740

Lyons, K., \& Wearing, S. (2008). Volunteer tourism as alternative tourism: Journeys beyond otherness. Journeys of discovery in volunteer tourism: International case study perspectives, LIVRO, 3-11.

Marcoux, J. S. (2009 December). Escaping the gift economy. Journal of Consumer Research, Oxford, 36(4), 671-685.

Maxwell, N. (1987 August). From knowledge to wisdom: A revolution in the aims and methods of science. Publisher: Blackwell Pub.

Maxwell, N. (2007 July). From knowledge to wisdom: the need for an academic revolution. London Review of Education, London, 5(2), 97-115.

McGehee, N. G. (2014 April). Volunteer tourism: evolution, issues and futures. Journal of Sustainable Tourism, London, UK, 22(6), 847854.

McGehee, N. G., \& Andereck, K. (2009 January). Volunteer tourism and the "voluntoured": the case of Tijuana, Mexico. Journal of Sustainable Tourism, London, UK, 17(1), 39-51.

McGehee, N. G., \& Santos, C. A. (2005 July). Social change, discourse and volunteer tourism. Annals of Tourism Research, Urbana, Illinois, United States 32(3), 760-779.

McIntosh, A. J., \& Zahra, A. (2007 January). A cultural encounter through volunteer tourism: Towards the ideals of sustainable tourism? Journal of Sustainable Tourism, London, UK, 15(5), 541-556. 
Mertens, D. M. (2007 April). Transformative paradigm mixed methods and social justice. Journal of mixed methods research, Newcastle,UK 1(3), 212-225.

Mertens, D. M. (2010). Transformative mixed methods research. Qualitative inquiry. Newcastle,UK: SAGE

Mick, D. G. (2006). Meaning and mattering through transformative consumer research. Advances in consumer research, Duluth, MN, 33(1), 1-4.

Mick, D. G., Pettigrew, S., Pechmann, C., \& Ozanne, J. L. (2012). Origins, qualities, and envisionments of transformative consumer research. Transformative consumer research for personal and collective wellbeing, New York, NY: Routledge, 3-24.

Ozanne, J. L. (2011). Introduction to the special issue on transformative consumer research: Creating dialogical spaces for policy and action research. Journal of Public Policy \& Marketing, Chicago, 30(1), 1-4.

Ozanne, J. L., \& Anderson, L. (2010). Community action research. Journal of Public Policy \& Marketing, Chicago 29(1), 123-137.

Ozanne, J. L., \& Fischer, E. (2012). Sensitizing principles and practices central to social change methodologies. Transformative consumer research: For personal and collective well-being, New York, NY: Routledge, 89-106.

Ozanne, J. L., \& Saatcioglu, B. (2008 Octuber). Participatory action research. Journal of Consumer Research, Oxford, 35(3), 423-439.

Rattan, J. K., Eagles, P. F., \& Mair, H. L. (2012 August). Volunteer tourism: its role in creating conservation awareness. Journal of Ecotourism, New York, NY: Routledge 11(1), 1-15.

Rosenbaum, M. S., Corus, C., Ostrom, A. L., Anderson, L., Fisk, R. P., Gallan, A. S., . . . Rayburn, S. W. (2011). Conceptualisation and aspirations of transformative service research. Journal of Research for Consumers, Oxford, 19, 1-6. 
Shultz, C. J., \& Shapiro, I. S. J. (2012). Transformative Consumer Research in Developing Economies. Transformative consumer research for personal and collective well-being, New York, NY: Routledge, 131.

Sin, H. L. (2009 July). Volunteer tourism—“involve me and I will learn"? Annals of Tourism Research, Guildford, UK, 36(3), 480-501.

Speth, J. G. (2008). The Bridge at the Edge of the World. New Haven: Yale.

Stoddart, H., \& Rogerson, C. M. (2004 July). Volunteer tourism: The case of habitat for humanity South Africa. GeoJournal, localNão tem, 60(3), 311-318.

Wearing, S. (2001). Volunteer tourism: Experiences that make a difference, Wallingford: CABI Publishing.

Wearing, S., \& McGehee, N. G. (2013 Octuber). Volunteer tourism: A review. Tourism Management, New York, NY 38, n. xxNão possui., 120-130.

Wearing, S., McDonald, M., \& Ponting, J. (2005 January). Building a decommodified research paradigm in tourism: The contribution of NGOs. Journal of Sustainable Tourism, local, 13(5), 424-439.

Wilkie, W. L., \& Moore, E. S. (1999). Marketing's contributions to society. The Journal of Marketing, Chicago, v.63, n.4, 198-218.

Wilkie, W. L., \& Moore, E. S. (2003). Scholarly research in marketing: Exploring the " 4 eras" of thought development. Journal of Public Policy \& Marketing, Chicago, 22(2), 116-146.

Wilkie, W. L., \& Moore, E. S. (2012). Expanding our understanding of marketing in society. Journal of the Academy of Marketing Science, Seattle, WA , 40(1), 53-73.

Artigo recebido em: 11/09/2015

Aprovado em: 18/12/2015 